\title{
Influence of wall material on nucleate pool boiling of liquid nitrogen
}

\author{
C. Bombardieri, C. Manfletti \\ Institute of Space Propulsion - German Aerospace Center (DLR), 74239 Hardthausen, \\ Germany \\ E-mail: cristiano.bombardieri@dlr.de
}

\begin{abstract}
The wall material thermal conductivity $k$ is one of the surface characteristics that influences the pool boiling heat transfer coefficient (HTC). It is believed that growth rates of vapor bubbles and their frequency are mechanisms affected by the thermal conductivity. In order to investigate this effect, different surface materials (copper, aluminum, stainless steel) are tested in a horizontal surface geometry. From a physical point of view, the redistribution of local heat fluxes between the free surface (wet by the liquid) and the spot covered with nucleation centers is hindered in the case of low values of $k$. But the wall material $k$ affects the nucleate boiling curve only in the case of a very limited number of nucleation sites. Previous experiments in fact concluded that for a rough surface that has a larger number of vapor generating centers, the wall thermal conductivity does not significantly affect the boiling HTC; therefore the tested surfaces are mechanically polished to avoid any roughness effect.

In this paper, nucleate pool boiling curves of saturated nitrogen under ambient pressure are experimentally obtained on a horizontal flat surface and the effect of three different wall materials is evaluated. Hysteresis behaviour has been observed for the copper and aluminum surfaces, while the results for stainless steel are contradictory. The critical heat flux (CHF) is also compared and analyzed by taking into account the thermal effusivity of the different materials. Finally, experimental results are compared against the most common empirical correlations for nucleate pool boiling heat transfer.
\end{abstract}

\section{Introduction}

Interest in boiling heat transfer of cryogenic fluids such as hydrogen, nitrogen, oxygen dates back to ' 50 and ' 60 due to the growing number of space applications. The applications where cryogenic boiling occurs are numerous: chill-down of cryogenic tanks and pipelines, thermal control system of satellites, cryogenic phase-change heat exchanger, production and use of cryogenics and their related hardware (liquifier, cryo-cooler, vaporizer).

Pool boiling heat transfer is a subject of extensive researches by many scientists worldwide. A large number of both theoretical and empirical correlations have been proposed to predict the pool boiling heat transfer coefficient (HTC) in the last 80 years, and this gives an idea of the challenge to correctly model the boiling phenomenon. The nucleate pool boiling curve is dependent on many parameters, mainly the thermophysical properties of the boiling fluid and of the surface. In particular the effects of the surface characteristics such as thermophysical properties of the material, geometry, thickness, orientation, surface conditions, roughness, etc., still require further investigation [1]. Among the thermophysical properties of the surface material, thermal conductivity $k$ and thermal effusivity $\varepsilon$ play an important role. In his work, Pioro [2] performed 
several experiments in order to evaluate the constants for the Rohsenow boiling correlation for different fluid-material combinations (water, ethanol, R-11, R-113 with copper, aluminum, brass, stainless steel). He remarked the differences among the tested material, which are indeed taken into account in the value of the constants, however the slope of the boiling curve $-q \propto \Delta T^{n_{-}}$ was not affected by the material. Numerical simulation performed by Mann et al. [3] showed that the influence of the wall thermal conductivity is small due to two compensating effects: a lower value of $k$ decreases the local heat flux, but it also leads to thinner liquid films in the micro region which enhances heat conduction in the film and causes higher heat fluxes.

Another aspect to be considered is the effect of the surface roughness. A rough surface provides an high number of nucleation sites and therefore the influence of the wall thermal conductivity is less significant. However for the boiling of cryogenic fluids, the wall thermal conductivity affects the HTC even with moderate level of roughness. Cryogenic fluids in fact wet the surface very well because their contact angles are close to 0 and therefore a surface that is rough for normal fluids can be considered smooth for cryogenics. Nucleate pool boiling of cryogenic liquids from ambient to near the critical pressure has been investigated by Kosky and Lyon [4] for oxygen, nitrogen, argon, methane and carbon tetrafluoride; by Bewilogua et al. [5] for helium, hydrogen, and nitrogen. The maximum value of the critical heat flux (CHF) was found at about $35 \%$ of the critical pressure.

The influence of the surface material on the HTC has been investigated by several authors $[3,6,7,8,9]$ mainly with water and refrigerants, but very few studies for liquid nitrogen are available. Ackermann et al. [10] performed pool boiling experiments of nitrogen with copper, German silver and aluminum surfaces under ambient pressure. No remarkable difference in the pool boiling curves has been noticed.

This paper presents the experimental pool boiling curves of saturated liquid nitrogen at ambient pressure on different surface material, namely copper, aluminum and stainless steel. Tested surface is a horizontal flat plate mechanically polished.

\section{Experimental set-up}

\subsection{Apparatus}

The apparatus used for the pool boiling experiments consists of a boiling vessel at the bottom of which the heating surface is mounted (Fig. 1). The boiling vessel is an insulated stainless steel box, base 180x120 mm and $320 \mathrm{~mm}$ in height. The vessel features a quartz window for visual investigation of the boiling phenomenon, however video imaging results are not not discussed here. The heating surface is a $8 \mathrm{~mm}$ thick plate, size $46 \times 51 \mathrm{~mm}$, mounted on a $20 \mathrm{~mm}$ thick copper block. The copper block is heated at the bottom by a ceramic heater connected to an electrical power supply with output voltage up to $150 \mathrm{VDC}$, maximum current $20 \mathrm{~A}$ for a maximum power of $3000 \mathrm{~W}$. The ceramic heater is a high temperature-pressed $S i_{3} N_{4}$ material and can withstand temperature up to $1300 \mathrm{~K}$. The $20 \mathrm{~mm}$ thick copper block is used for heat flux measurement as explained in Section 2.3. Inside the copper block six thermocouples are mounted in two parallel rows (Fig. 2), the distance between the thermocouple is $15 \mathrm{~mm}$ horizontal and 12 $\mathrm{mm}$ between the rows. Onto the copper block the test element of the selected material is placed. It is a $8 \mathrm{~mm}$ thick plate with three thermocouples located $4 \mathrm{~mm}$ beneath the surface. In this way measurements of temperature in three different position are possible (left, middle, right) so that the heat flux uniformity can be evaluated. The test elements are made of copper, aluminum and stainless steel 304L. They are mechanically polished in order to avoid any roughness effect.

The heating power is provided by setting the VDC supply regulator by means of stepwise increases (and decreases). 

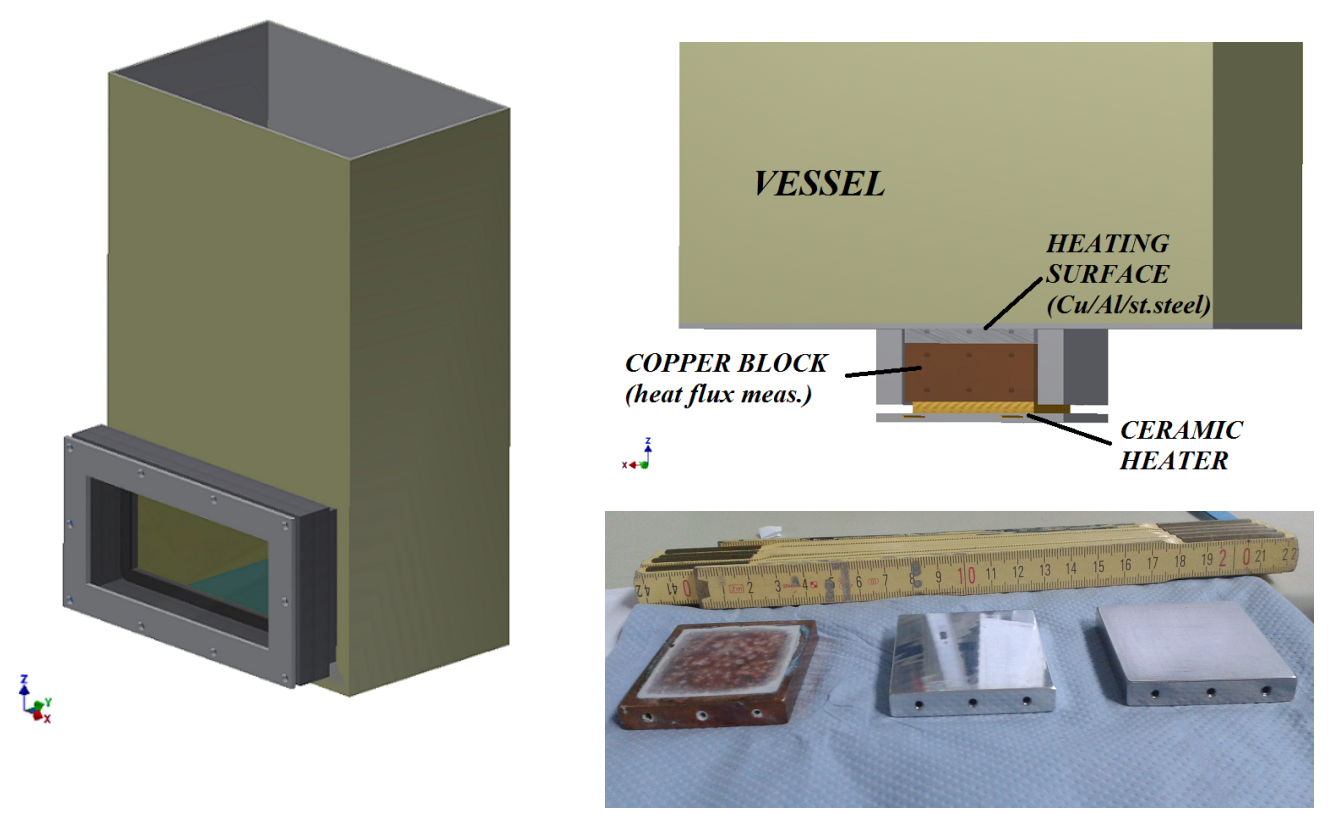

Figure 1. Experimental apparatus: vessel (left) and details of the heating block (right). Right bottom: the three different heating surfaces used, copper, aluminum, stainless steel 304L
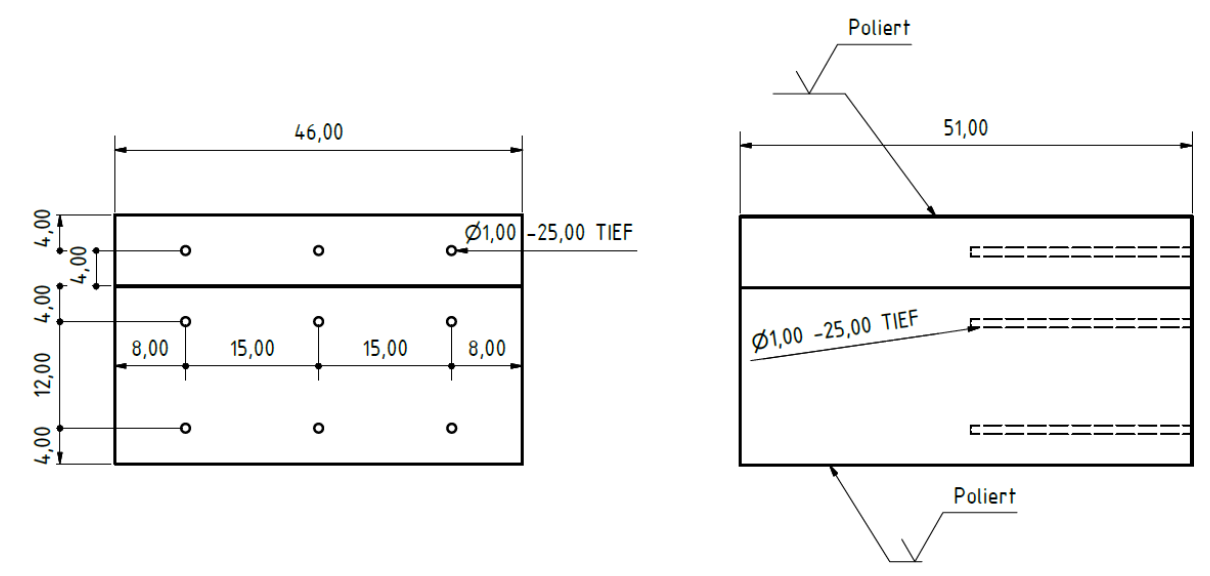

Figure 2. Drawing of the copper block and the test element surface (on top of it)

\subsection{Thermocouples and data acquisition}

Thermocouples used are type K, $0.5 \mathrm{~mm}$ tip with stainless steel sheath. An ice bath box is used for the cold junction reference temperature. Each thermocouple signal is amplified (gain = 1000) and filtered (low pass filter set at $200 \mathrm{~Hz}$ ). The conditioned signals are sampled at $1 \mathrm{KHz}$ with a National Instrument acquisition card NI-PXI-6289 at 18 bit. The thermocouples are calibrated in liquid nitrogen in situ and then the signal is corrected by applying the two-points calibration method according to Suslov [11]. This method takes into account the non-linearity of the voltage error difference outside the given calibration points. Instead of a polynomial interpolation, the NIST look-up table is directly used for the conversion mv to degree C. At each heat power level, temperature signals are recorded for 8 seconds and then the averaged value is used. 


\subsection{Calculation of heat flux and surface temperature}

The surface temperature $T_{s}$ is calculated from the measured average temperature in the test element insert $\left(T_{w}\right)$ and the heat flux $q$ by applying the heat conduction equation:

$$
T_{s}=T_{w}-q \frac{\triangle x}{k_{w}}
$$

where $\triangle x$ is the distance to the surface $(4 \mathrm{~mm})$ and $k_{w}$ is the wall material thermal conductivity.

The heat flux $q$ is calculated in the copper block by $q=k_{C u} \frac{\Delta T_{C u}}{\Delta x_{c u}}$, with $\triangle T_{C u}$ the measured temperature difference and $\triangle x_{C u}$ the vertical distance of the thermocouples $(12 \mathrm{~mm})$. The calculated heat flux is compared to the heating power from the voltage supply regulator. In almost all the test the calculated heat flux is between $93 \%$ and $98 \%$ of the provided power. In some test run a value of $102 \%$ was calculated: in this case the heat flux is corrected to $98 \%$. Values of heat flux less than $93 \%$ and over $102 \%$ are rejected. The reason for this deviation is due to the tolerances in the placement of the thermocouples, the thermocouple measurement errors and the inaccuracy of the value of the thermal conductivity at cryogenic temperature. Although the heat flux value could be taken from the power supply directly, the uncertainty on the heat losses would not recommended this options. A detailed error analysis is presented in the next section.

\subsection{Uncertainties analysis}

To evaluate the uncertainties on the measurements, the procedure recommended in [12] is adopted. Since the heat flux $q$ is not measured directly, the law of propagation of uncertainty (Eq. 2) is used:

$$
f_{U}=\sqrt{\sum\left(\frac{\partial f}{\partial x_{i}} x_{i, U}\right)^{2}}
$$

where $f_{U}$ is the uncertainty of the dependent variable and $x_{i, U}$ the uncertainty of the independent variables. The error on the heat flux is therefore evaluated by Eq. (3):

$$
\frac{q_{U}}{q}=\sqrt{\left(\frac{\triangle x_{U}}{\triangle x}\right)^{2}+\left(\frac{k_{U}}{k_{C u}}\right)^{2}+\left(\frac{\triangle T_{U}}{\triangle T_{C u}}\right)^{2}}
$$

where $\triangle x$ is the distance between the thermocouple (12 $\mathrm{mm}), k_{C u}$ is the copper thermal conductivity, $\triangle T_{C u}$ the measured temperature difference. The error on $T_{s}$, calculated as in Eq. $(1)$, is then

$$
T_{s, U}=\sqrt{\left(T_{w, U}\right)^{2}+\left(q \frac{\triangle x_{w}}{k_{w}}\right)^{2}\left[\left(\frac{\triangle x_{U}}{\triangle x_{w}}\right)^{2}+\left(\frac{k_{w U}}{k_{w}}\right)^{2}+\left(\frac{q_{U}}{q}\right)^{2}\right]}
$$

where $\triangle x_{w}$ is the distance to the surface ( $4 \mathrm{~mm}$ ), $k_{W}$ the surface material (copper, aluminum, stainless steel) thermal conductivity, $q$ the heat flux calculated in the copper block.

Since the thermocouples are calibrated in liquid nitrogen $(77 \mathrm{~K})$, the systematic error is practically eliminated. However, in the near range of the calibration $(77-110 \mathrm{~K})$, the error can be estimated in $0.2 \mathrm{~K}$. The tolerance in the placement of the thermocouples is $0.05 \mathrm{~mm}$. The value of the thermal conductivity $k$ is also affected by uncertainty. At cryogenic temperature its sensitivity on the temperature is high and must be taken into account. In the present work, values of $k$ for the used materials are taken from NIST, however others references $[13,14]$ indicate different values in the cryogenic range. The uncertainty of $k$ is then considered as $5 \%$. 
The overall uncertainties on the heat flux $q$ and wall temperature $T_{s}$ are summarized in Table 1 for a low and an high heat flux level, namely the critical heat flux (CHF).

Table 1. Uncertainty of the calculated value $q$ and $T_{s}$ for the different materials at lox heat flux (25\% of $\mathrm{CHF}$ ) and at $\mathrm{CHF}$

\begin{tabular}{lccc}
\hline & copper & aluminum & st. steel \\
\hline$q_{\text {err }}$ at $25 \%$ CHF [\%] & 27 & 43 & 47 \\
$q_{e r r}$ at CHF [\%] & 8.3 & 12 & 13 \\
$T_{s, e r r}$ at $25 \%$ CHF [K] & 0.22 & 0.27 & 4.1 \\
$T_{s, e r r}$ at CHF [K] & 0.24 & 0.29 & 4.4
\end{tabular}

The high error on $T_{s}$ for stainless steel is due to the high temperature drop in the test element (due to its low $k$ ) combined with the uncertainty of the heat flux. In fact the uncertainty on $T_{s}$ can be written as follows:

$$
T_{s U} \approx\left(T_{s}-T_{w}\right) \cdot \frac{q_{U}}{q}=\frac{\triangle x_{w}}{k_{w}} q_{U}
$$

In order to improve the accuracy, the error on the heat flux $q_{U}$ must be decrease (e.g. by increasing the distance of the thermocouples in the copper block). However, the calculated heat flux is always compared to the one from the power supply so that its accuracy is actually much higher (the power supply has an accuracy of less than $0.5 \%$ ). Moreover, since the value of the heat flux is corrected by evaluating its ratio with the power supply (rejected if higher than 102\%), it is assured that the value of $q$ is never over-estimated.

\subsection{Test Procedure}

Once the vessel is cooled down at cryogenic temperature, the liquid nitrogen is left $1 \mathrm{~h}$ at low boiling conditions to assure no gas is dissolved and thermal steady state is reached. The heat flux is stepwise increased from 0 up to the critical heat flux (CHF) by setting the voltage supply regulator. At low heat flux the increments are $\sim 1 W / \mathrm{cm}^{2}$ while a more refined value is used when approaching the CHF. The down-reading $q \downarrow$ with the same decrease is also performed to evaluate hysteresis effects. At every heating power level, a 6 minutes time is awaited in order to ensure that steady condition is achieved before recording the signal. Experimentally, the temperature reaches constant value after $2 \mathrm{~min}$, anyway an additional $4 \mathrm{~min}$ waiting time is added as a precaution. In the case of stainless steel, the waiting time is increased to 8 minutes.

\section{Results and discussion}

\subsection{Effect of the material}

The effect of the material properties has been investigated in the past for fluids such as water and refrigerants $[2,3,6,7,8,9]$. It has been recognized that material does affect the pool boiling heat transfer, though it is difficult to take this effect into account into usable correlations. Rohsenow (1952) first introduced an empirical ad hoc coefficient $C_{s f}$ to include the effect of different surfacefluid combinations (see Section 3.3). As a general statement, an higher wall material thermal conductivity $k$ increases the heat transfer for the same wall temperature, if the surface is not too rough. From a physical point of view, the redistribution of local heat fluxes between the free surface (wet with liquid) and the spot covered with nucleation centers is hindered in the case of low values of $k$. However the wall material $k$ affects the nucleate boiling curve only 
in the case of a very limited number of potential vapor generating centers, e.g. in the case of a polished surface. For a rough surface that has a high number of nucleation sites, the wall thermal conductivity does not significantly affect the boiling heat transfer [1]. Jabardo et al. [6] investigated the boiling of fluorocarbon refrigerants R-134a and R-123 on copper, brass and stainless steel surfaces. Results of their experiments show that the slope of the curve HTC vs $q$ is affected by the surface material. They also suggested the influence of a combined surface-liquid effect instead of an isolated surface material effect.

The effect of the surface thermophysical properties can be expressed in the form of a ratio of the thermal effusivity $\varepsilon$ of the surface material to that of the liquid:

$$
\Lambda=\sqrt{\frac{\left(\rho k c_{P}\right)_{w}}{\left(\rho k c_{P}\right)_{f}}}
$$

For non-cryogenic fluids, the HTC in nucleate boiling regime is dependent on this ratio as $h \propto \Lambda^{0.4}$ [15], but it can have a even more pronounced dependency ( $h \propto \Lambda^{0.5}$ according to [13]).

Stephan and Abdelsalam [15] performed a regression analysis on more than five thousand data points from seventy-two experiments. They proposed that the data could be best represented by dividing the liquids in four groups. For the case of cryogenic fluids, they proposed the following relationship to take into account the material properties :

$$
h=C_{1} q^{0.624}\left(\rho k c_{P}\right)_{w}^{0.117}=C_{1} q^{0.624} \varepsilon^{0.234}
$$

Since $h=\frac{q}{\Delta T}$, rearranging Eq. (6) in the most common relationship $q(\triangle T)$, leads to:

$$
q=C_{2} \Lambda^{0.622} \triangle T^{2.66}
$$

where the constant $C_{2}$ includes the thermal effusivity of the fluid $\varepsilon_{f}$ so that the ratio $\Lambda$ can be written explicitly. Table 2 displays the values of the thermal effusivity ratio for copper, aluminum and stainless steel 304L with respect to water and liquid nitrogen and the heat flux ratio calculated with respect to copper.

Table 2. Values of the thermal effusivity $\varepsilon$ for water and nitrogen at saturation pressure of 1 bar and thermal effusivity ratio for copper, aluminium and stainless steel 304L. The heat flux ratio of aluminum and stainless steel with respect to copper is calculated by assuming a dependency $q \propto \Lambda^{0.662}$ as proposed by [15].

\begin{tabular}{ccccccc}
\hline Fluid & $\begin{array}{c}\varepsilon=\sqrt{\rho k c_{P}} \\
{\left[W s^{0.5} / m^{2} / K\right]}\end{array}$ & $\Lambda_{\text {copper }}$ & $\Lambda_{\text {alu }}$ & $\begin{array}{c}q_{A l} / q_{C u} \\
\propto \Lambda^{0.622}\end{array}$ & $\Lambda_{\text {st.steel }}$ & $\begin{array}{c}q_{s s} / q_{C u} \\
\propto \Lambda^{0.622}\end{array}$ \\
\hline water & 1656 & 22.5 & 14.5 & 0.76 & 4.68 & 0.38 \\
LN2 & 489 & 67.8 & 40.7 & 0.73 & 9.24 & 0.29
\end{tabular}

Applying Eq. (6) to liquid nitrogen, the aluminum and stainless steel give respectively a heat flux $73 \%$ and $29 \%$ of the value of copper .

Fig. 3 shows the experimental results obtained for the three different surface materials tested together with a fitting curve based on a power law $q=C_{1} \triangle T^{n}$. For the fitting curve only the heat fluxes calculated at the middle of the test surface are used, being the side heat fluxes used only for the verification of the heat losses. An example of the side heat fluxes is shown for copper (top, left). In the case of the stainless steel surface, only few data points have been used for the analysis: many data have been rejected since the calculated heat flux did not match the one from the power supply as explained in Section 2.3. 

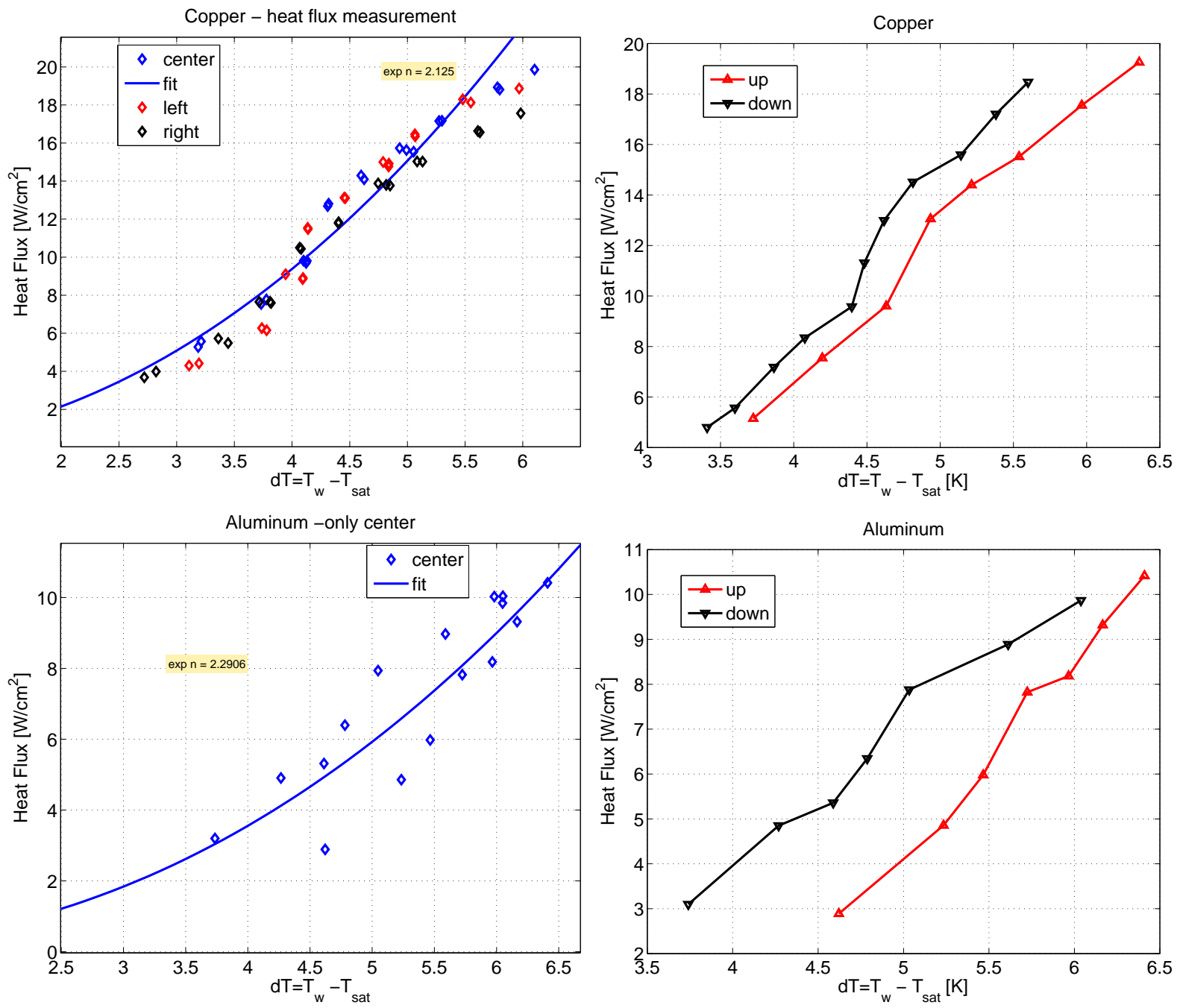

St. Steel -only center
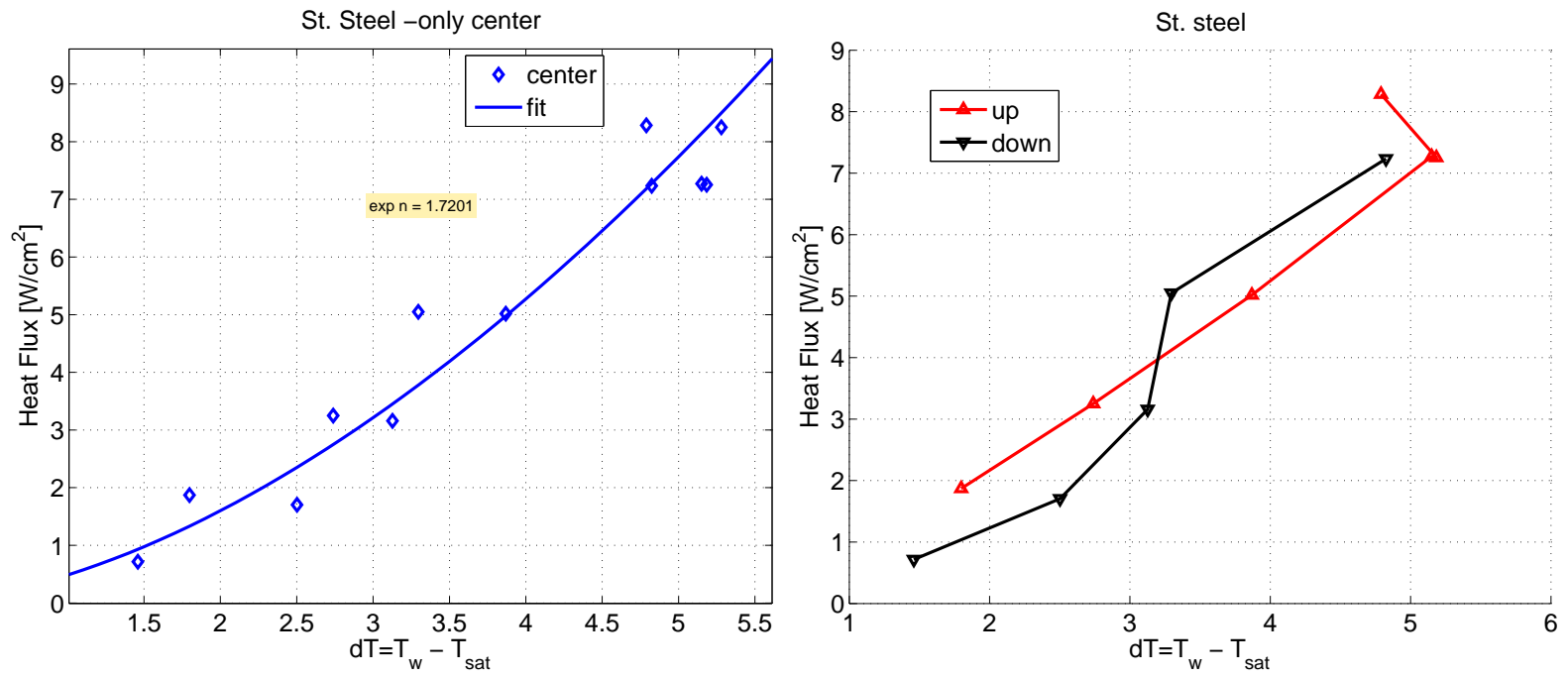

Figure 3. Experimental data of nucleate pool boiling for copper (top), aluminum (middle), stainless steel (bottom). The fitting curves assuming a power law $q=C_{1} \triangle T^{n}$ are also plotted. Hysteresis behaviour is showed on the right. 


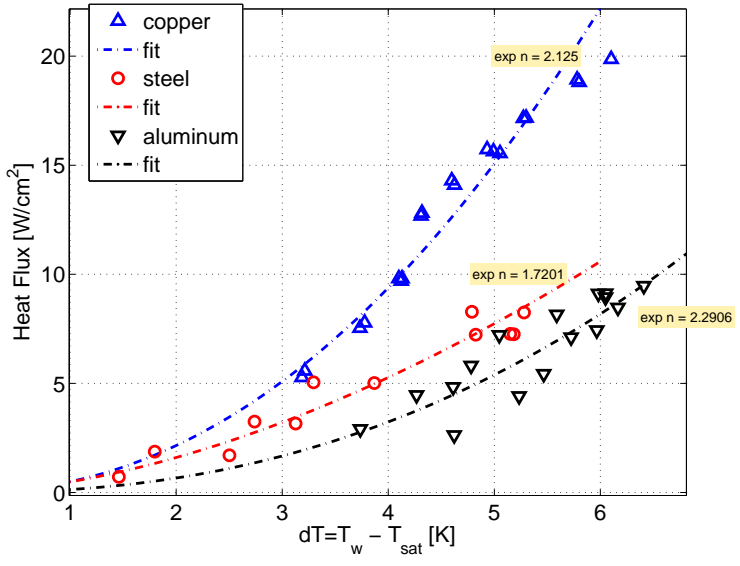

Figure 4. Comparison of the experimental data for the different tested materials

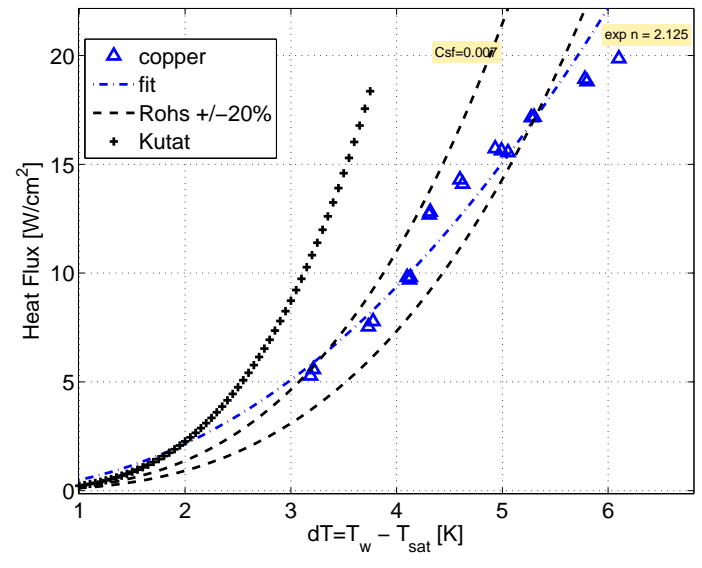

Figure 5. Comparison of the copper data with the nucleate pool boiling correlations of Rohsenow -Eq.(8)- and Kutateladze -Eq.(9)

A comparison among the experimental results for the different materials is showed in Fig. 4. The slope $n$ of the nucleate boiling curve $q=C_{1} \triangle T^{n}$ is lower than the well-known value of 3 for all the tested material, though $n=2.66$ could be used instead as a reference for cryogenic fluids (see Eq. 7). The slope is highest for aluminum $(n=2.29)$ and lowest for stainless steel $(n=1.72)$, although it is questionable the sense of the slope index at such a low temperature difference. The value of the coefficient $C_{1}$ has a larger weight than the slope index $n$ on the heat flux $q$, in fact the values of the heat flux for copper are higher than the aluminum ones despite a lower $n$ (2.125 for copper). The results for stainless steel should be considered carefully, because of the high error affecting the measurements. From an experimental point of view, results for copper are more consistent than the other materials and showed a better reproducibility.

\subsection{Hysteresis}

Because boiling is an inherently non-equilibrium process, it is reasonable to assume it involves some hysteresis behavior. Hysteresis in pool boiling of cryogenic fluid was observed by Kosky and Lyon [4]. In their experiments they used an horizontal platinum surface and they noticed an inverted hysteresis loop at intermediate and high saturation pressure for oxygen, argon and methane.

In the present work, hysteresis effects have been observed for copper and aluminum, while no conclusion can be drawn for stainless steel due to the high uncertainty of its results. Copper shows a weak hysteresis effect (Fig. 3, top right ). The temperature difference between the increasing heat flux curve and the decreasing one is less than $0.3 \mathrm{~K}$ at the same heat flux value. The hysteresis is more remarked in the case of aluminum (Fig. 3, middle right), while the results of stainless steel (Fig. 3, bottom right) are contradictory, being the decreasing curve $q \downarrow$ greater than the increasing one $q \uparrow$ at higher heat flux (as in the other cases) and the opposite at low value of $q$.

\subsection{Comparison with nucleate pool boiling correlations}

Many empirical correlations have been proposed for the nucleate pool boiling regime. The first and most famous correlation is due to Rohsenow (Eq. 8). It is based on a dimension analysis of the involved variables and it takes into account the liquid-surface combination effects by an ad $h o c$ coefficient, $C_{s f}$. 


$$
\frac{c_{p f} \triangle T_{s a t}}{h_{f g}}=C_{s f}\left[\frac{q}{h_{f g} \mu_{f}}\left(\frac{\sigma}{g\left(\rho_{f}-\rho_{g}\right)}\right)^{1 / 2}\right]^{r} \operatorname{Pr}_{f}^{s}
$$

The coefficient $C_{s f}$ includes material and surface finishing effects, such as surface roughness. Rohsenow indicated different values of $C_{S f}$ between 0.0025 and 0.013 for various fluid-surface combination. The exponents $r$ and $s$ take into account the pressure effects, typically $r=0.33$ and $s=0.8 \doteqdot 2$ (original value was 1 for water and 1.7 for other fluids). If the heat flux is expressed as a function of the temperature difference $\triangle T$, the equation assumes the most known form $q \propto \Delta T^{3}$. An experimental evaluation of the value of $C_{s f}$ for several fluid-surface combinations is provided by Pioro [2]. According to his work, for all the tested fluid-surface combinations (water/ethanol/R-11/R-113 with copper/aluminum/brass/steel) an exponent $r=0.33$ fits fairly well all the data, while $C_{s f}$ varies considerably (e.g. 0.006 for water-steel and 0.0147 for watercopper polished). No data are provided for cryogenic fluids. In [14] a value of $C_{s}=0.013$ is indicated for nitrogen. For cryogenics, the Kutateladze correlation (Eq. 9) has been verified for oxygen, nitrogen, hydrogen, helium [14]:

$$
\frac{c_{p f} \triangle T_{s a t}}{h_{f g}}=0.0007\left[\frac{q}{h_{f g} \mu_{f}}\left(\frac{\sigma}{g\left(\rho_{f}-\rho_{g}\right)}\right)^{1 / 2}\right]^{0.3} \operatorname{Pr}_{f}^{0.65}\left[\frac{\rho_{g}}{\rho_{f}} \frac{P_{s a t}}{\sqrt{g \sigma\left(\rho_{f}-\rho_{g}\right)}}\right]^{0.7}
$$

Fig. 5 compares the experimental data obtained for copper with Rohsenow's and Kutateladze's correlations. For Rohsenow's correlation the value of $C_{s f}$ has been adjusted to 0.007 in order to better fit the data. The experimental data obtained with copper are within $\pm 20 \%$ of the predictive Rohsenow's curve, except at high heat flux, although the original slope index $n=3$ is kept while its fitted value $n=2.125$ would be better. Kutateladze's correlation is not matching the experimental data at all. One should note that Kutateladze's correlations does not include any material effect.

Critical heat flux In addition to nucleate boiling curves, measurements of the critical heat flux (CHF) are taken as well. Table 3 summarizes the results. The error on the CHF is evaluated in about $8.3 \%, 12 \%, 13 \%$ for copper, aluminum and stainless steel respectively.

Table 3. Values of the experimental CHF obtained for the different tested materials

\begin{tabular}{lccc}
\hline & copper & aluminum & st.steel \\
\hline $\mathrm{CHF}\left[W / \mathrm{cm}^{2}\right]$ & 19.5 & 11.5 & 8.3 \\
$\triangle T[K]$ & 6.8 & 6.4 & 5.2
\end{tabular}

For the prediction of the CHF, Lienhard, Dhir and Riherd [16] developed the following relationship for flat plate heaters:

$$
q_{\max }=0.1492 \rho_{g} h_{f g}\left[\frac{g \sigma_{L}\left(\rho_{f}-\rho_{g}\right)}{\rho_{g}^{2}}\right]^{0.25}
$$

In the case of liquid nitrogen, Eq. (10) gives a CHF of $18.3 \mathrm{~W} / \mathrm{cm}^{2}$ very close to the value obtained with the copper surface (-6\% difference). Since Eq. (10) does not consider any material effect, Eq. (7) could be applied to estimated the CHF for other materials than copper.

The CHF of the aluminum surface is $59 \%$ of the value obtained with the copper surface, in contrast with the predicted value of $73 \%$ given by Eq. (7); the CHF of stainless steel is $42 \%$ of the value of copper (predicted 29\%). The dependency of the CHF on the material properties 
is conflicting, being over-estimated for aluminum and under-estimated for stainless steel. This result indicates that the dependency relationship $q \propto \Lambda^{x}$ may be inadequate to correctly consider the influence of the wall thermophysical properties. The wall thermal conductivity in fact affects two important boiling mechanisms: the growth rates of vapor bubbles and the waiting time between two successive bubbles [3]. These two aspects are strongly non-linear and the power law relationship appears to be a simplified assumption not taking into account the complex physics of the boiling process.

\section{Conclusions}

Nucleate pool boiling curves of saturated liquid nitrogen on different surface materials are experimentally obtained. The results show that the material affects not only the heat flux value at a given wall superheat but also the slope of the boiling curve $q-\triangle T$. The highest slope index is obtained for aluminum $(n=2.29)$, while stainless steel showed the lowest value $(n=1.72)$. At the same wall temperature, results clearly indicate that copper has the highest heat fluxes, while surprisingly aluminum has lower heat fluxes than stainless steel. Nevertheless, results obtained for stainless steel are affected by a not negligible error.

The critical heat flux is also the highest for copper $\left(19.5 \mathrm{~W} / \mathrm{cm}^{2}\right)$, very closed to the predicted value of $18.3 \mathrm{~W} / \mathrm{cm}^{2}$ obtained with the most common correlation of Lienhard-Dhir-Riherd. The influence of the material thermo-physical properties is evaluated by taking into account the thermal effusivity ratio material-liquid $\Lambda$, however the experimental $\mathrm{CHF}$ of aluminum and stainless steel is conflicting with the prediction, being over-estimated for aluminum and underestimated for stainless steel. This may indicate that the dependency relationship $q \propto \Lambda^{x}$ is inadequate to correctly consider the influence of the wall material thermophysical properties.

Hysteresis effects have also been observed for copper and aluminum and they are in agreement with previous findings, being the curve during decreasing heat flux higher than the one for increasing heat flux. However the hysteresis behavior of stainless steel is contradictory.

A comparison of the experimental results with the nucleate pool boiling correlations of Rohsenow and Kutateladze shows that both correlations fail to predict the heat flux. Rohsenow's correlation matches the data within $\pm 20 \%$ only by fitting the fluid-surface coefficient.

Future experiments will be performed by using a rough surface with the same tested materials in order to evaluate the combined effect of material and roughness on nucleate pool boiling of liquid nitrogen.

\section{Reference}

[1] Pioro I, Rohsenow W and Doerffer S 2004 International Journal of Heat and Mass Transfer 475033 - 5044

[2] Pioro 1998 International Journal of Heat and Mass Transfer 422003 - 2013 ISSN 0017-9310

[3] Mann M, Stephan K and Stephan P 2000 International Journal of Heat and Mass Transfer 432193 - 2203

[4] Kosky P G and Lyon D N 1968 AIChE Journal 14 372-379 ISSN 1547-5905

[5] Bewilogua L, Knöner R and Vinzelberg H 1975 Cryogenics 15 121-125

[6] Jabardo J M S, Ribatski G and Stelute E 2009 Experimental Thermal and Fluid Science 33579 - 590

[7] Ma K T and Pan C 1999 International Communications in Heat and Mass Transfer 261103 - 1114

[8] Reza Hosseini Amir Gholaminejad M N 2011 Journal of Electronics Cooling and Thermal Control 1

[9] Benjamin R and Balakrishnan A 1997 Experimental Thermal and Fluid Science 1532 - 42 ISSN 0894-1777

[10] Ackermann H, Bewilogua L and Vinzelberg H 1975 Cryogenics 15

[11] Suslov 2006 Verfahren und vorrichtung zur temperaturbestimmung mittels eines thermoelements

[12] Taylor B N and Kuyatt C 1994 Guidelines for evaluating and expressing the uncertainty of nist measurement results Tech. rep. NIST

[13] VDI 2006 VDI-Wärmeatlas (Verein Deutscher Ingenieure, VDI-Gesellschaft Verfahrenstechnik und Chemieingenieurwesen (GVC))

[14] Barron R 1999 Cryogenic heat transfer Series in chemical and mechanical engineering (Taylor and Francis)

[15] Stephan K and Abdelsalam M 1980 International Journal of Heat and Mass Transfer 2373 - 87

[16] Lienhard J 2003 A heat transfer textbook (Phlogiston Press) 